\title{
Fractional order differential equations with iterations of linear modification of the argument
}

\author{
JinRong Wang ${ }^{1,2^{*}}$ and JianHua Deng ${ }^{2}$
}

\section{*Correspondence:}

wjr9668@126.com

${ }^{1}$ School of Mathematics and

Computer Science, Guizhou Normal

College, Guiyang, Guizhou 550018,

China

${ }^{2}$ Department of Mathematics,

Guizhou University, Guiyang,

Guizhou 550025, China

\begin{abstract}
In this paper, we apply Picard operator theory to investigate a class of fractional differential equations with iterations of linear modification of the argument. For that, two useful work spaces $C_{L}(J, J)$ and $C_{L, \theta}(J, J)$ with three powerful norms $\|\cdot\|_{B}\|\cdot\|_{\beta}$ and $\|\cdot\|_{C}$ are used, respectively. Some existence and uniqueness results are presented. Here, we introduce a new norm $\|\cdot\|_{\beta}$ and give another direct way to deal with the iterative term in the nonlinear term, which can be regarded as the main novelty in this paper.
\end{abstract}

MSC: 26A33; 34A12; 47H10

Keywords: fractional order differential equations; iterations; existence; Picard operator

\section{Introduction}

Integer order differential equations with iterations give a good approach to search for approximative solutions and have been discussed by many researchers [1-9] owning to their wide applications in engineer control and computational mathematics.

Recently, fractional order differential equations have appeared naturally in the fields such as viscoelasticity, electrical circuits, nonlinear oscillation of earthquake, etc. There are some remarkable monographs that provide the main theoretical tools for the qualitative analysis of fractional order differential equations and, at the same time, show the interconnection as well as the contrast between integer order differential models and fractional order differential models, [10-17].

In [9], the author discussed a first-order differential equation with iterations of linear modification of the argument

$$
\left\{\begin{array}{l}
x^{\prime}(t)=f(t, x(t), x(\lambda t), x(\lambda x(\lambda t))), \quad 0<\lambda<1, t \in J_{b}:=[0, b], b>0, \\
x(0)=0 .
\end{array}\right.
$$

The existence, existence and uniqueness, and data dependence for the solutions of equation (1) were analyzed by using Picard operators and weakly Picard operators methods. The importance of iterations of linear modification of the argument will help us to find a simple way and suitable parameter to find the solution.

O2013 Wang and Deng; licensee Springer. This is an Open Access article distributed under the terms of the Creative Commons Attribution License (http://creativecommons.org/licenses/by/2.0), which permits unrestricted use, distribution, and reproduction in any medium, provided the original work is properly cited. 
In [18], the authors extended to study fractional order case $q \in(0,1)$ :

$$
\left\{\begin{array}{l}
{ }^{c} D_{a, t}^{q} x(t)=f\left(t, x(t), x\left(x^{v}(t)\right)\right)+\lambda, \quad t \in[a, b], v \in R \backslash\{0\}, q \in(0,1), \lambda \in R, \\
x(t)=\varphi(t), \quad t \in\left[a_{1}, a\right], \\
x(t)=\psi(t), \quad t \in\left[b, b_{1}\right],
\end{array}\right.
$$

where ${ }^{c} D_{a, t}^{q}$ is the Caputo fractional derivative of order $q$ with the lower limit $a$ and $a_{1} \leq$ $a<b \leq b_{1}, a_{1} \leq a_{1}^{v}$ and $b_{1}^{v} \leq b_{1}, f \in C\left([a, b] \times\left[a_{1}, b_{1}\right]^{2}, R\right)$ and $\varphi \in C\left(\left[a_{1}, a\right],\left[a_{1}, b_{1}\right]\right)$ and $\psi \in C\left(\left[b, b_{1}\right],\left[a_{1}, b_{1}\right]\right)$.

Here, we extend to another fractional order case $q \in(1,2)$ :

$$
\left\{\begin{array}{l}
{ }^{c} D_{0, t}^{q} x(t)=f(t, x(t), x(\lambda t), x(\lambda x(\lambda t))), \quad 0<\lambda<1, t \in J:=[0,1], \\
x(0)=0, \quad x^{\prime}(0)=0,
\end{array}\right.
$$

where $1<q<2$ and $f$ is a Carathéodory function satisfying some assumptions that will be specified later. Clearly, equation (3) is a generalization of equations (1) and (2).

For the existence results of solutions for problem (3), we emphasize that the main difficulty from the fractional order derivative ${ }^{c} D_{0, t}^{q} x(\cdot)$ and iterative term $x(\lambda x(\lambda \cdot))$ in $f$. Compared with the results and methods in $[9,18]$, one can find that: (i) we introduce a new norm $\|\cdot\|_{\beta}$ and give another direct way to deal with the iterative term $x(\lambda x(\lambda \cdot))$ in $f$, which can be regarded as the main novelty in this paper; (ii) we derive new existence and uniqueness results for a general class of fractional order differential equations.

\section{Preliminaries}

We recall the definitions of fractional integrals and derivatives. For more details, one can refer to Kilbas et al. [12].

Definition 2.1 The fractional order integral of the function $h \in L^{1}(J, \mathbb{R})$ of order $q \in \mathbb{R}^{+}$is defined by

$$
I_{0, t}^{q} h(t)=\frac{1}{\Gamma(q)} \int_{0}^{t}(t-s)^{q-1} h(s) d s
$$

where $\Gamma$ is the gamma function.

Definition 2.2 For a function $h$ given on the interval $J$, the $q$ th Riemann-Liouville fractional order derivative of $h$ is defined by

$$
{ }^{L}\left(D_{0, t}^{q} h\right)(t)=\frac{1}{\Gamma(n-q)}\left(\frac{d}{d t}\right)^{n} \int_{0}^{t}(t-s)^{n-q-1} h(s) d s
$$

here $n=[q]+1$ and $[q]$ denotes the integer part of $q$.

Definition 2.3 The Caputo derivative of order $q$ for a function $f: J \rightarrow \mathbb{R}$ can be written as

$$
{ }^{c} D_{0, t}^{q} h(t)={ }^{L} D_{0, t}^{q}\left(h(t)-\sum_{k=0}^{n-1} \frac{t^{k}}{k !} h^{(k)}(0)\right), \quad t>0, n-1<q<n .
$$


Like in the proof of Lemma 3.1 in [19], one can see that a function $x \in C(J, J)$ given by

$$
x(t)=\frac{1}{\Gamma(q)} \int_{0}^{t}(t-s)^{q-1} h(s) d s
$$

is a unique solution of the following problem:

$$
\left\{\begin{array}{l}
{ }^{c} D_{0, t}^{q} x(t)=h(t), \quad t \in J, 1<q<2, \\
x(0)=0, \quad x^{\prime}(0)=0,
\end{array}\right.
$$

where $h \in C(J, J)$.

Next, we collect some notions and results from the weakly Picard operator theory (for more details, see Rus [20, 21]).

Let $(X, d)$ be a metric space, $A: X \rightarrow X$ be an operator, and $F_{A}=\{x \in X: A(x)=x\}$ be the fixed point set of $A$.

Definition 2.4 (Rus $[22,23])$ Let $(X, d)$ be a metric space. An operator $A: X \rightarrow X$ is a Picard operator if there exists $x^{*} \in X$ such that $F_{A}=\left\{x^{*}\right\}$ and the sequence $\left(A^{n}\left(x_{0}\right)\right)_{n \in \mathbb{N}}$ converges to $x^{*}$ for all $x_{0} \in X$.

Definition 2.5 (Jung et al. [24]) Suppose that $E$ is a vector space over $\mathbb{K}$. A function $\|\cdot\|_{\beta}(0<\beta \leq 1): E \rightarrow[0, \infty)$ is called a $\beta$-norm if and only if it satisfies (i) $\|x\|_{\beta}=0$ if and only if $x=0$; (ii) $\|\lambda x\|_{\beta}=|\lambda|^{\beta}\|x\|_{\beta}$ for all $\lambda \in \mathbb{K}$ and all $x \in E$; (iii) $\|x+y\|_{\beta} \leq\|x\|_{\beta}+\|y\|_{\beta}$.

Let $C(J, J)$ be the space of all continuous functions from $J$ into $J$. For some $L>0$ and $0<\theta \leq 1$, we consider the following spaces:

$$
\begin{aligned}
& C_{L}(J, J):=\left\{x \in C(J, J):\left|x\left(t_{1}\right)-x\left(t_{2}\right)\right| \leq L\left|t_{1}-t_{2}\right| \text { for all } t_{1}, t_{2} \in J\right\}, \\
& C_{L, \theta}(J, J):=\left\{x \in C_{L}(J, J): x(t) \leq \theta t \text { for all } t \in J\right\} .
\end{aligned}
$$

Meanwhile, we introduce three powerful norms $\|\cdot\|_{B},\|\cdot\|_{\beta}$ and $\|\cdot\|_{C}$ in the space of $C(J, \mathbb{R})$ which are defined by

$$
\begin{aligned}
&\|x\|_{B}:=\max _{t \in J}|x(t)| e^{-\tau t} \quad(\tau>0), \quad\|x\|_{\beta}:=\max _{t \in J}|x(t)|^{\beta} \quad(0<\beta<1), \\
&\|x\|_{C}:=\max _{t \in J}|x(t)| .
\end{aligned}
$$

Let $d_{B}, d_{\beta}$ and $d_{C}$ be their corresponding metrics, respectively.

Obviously, if $d \in\left\{d_{C}, d_{B}, d_{\beta}\right\}$, then the spaces $\left(C_{L}(J, J), d\right),\left(C_{L}^{q}(J, J), d\right)$ and $\left(C_{L, \theta}(J, J), d\right)$ are complete metric spaces.

\section{First results in $\left(C_{L, \theta}(J, J),\|\cdot\|_{B}\right)$}

We will use $\|\phi\|_{L^{p}(J)}$ to denote the $L^{p}\left(J, \mathbb{R}_{+}\right)$norm of $\phi$ whenever $\phi \in L^{p}\left(J, \mathbb{R}_{+}\right)$for some $p$ with $1<p<\infty$. Let $q_{i} \in(0,1), i=1,2$, and $\eta(\cdot) \in L^{\frac{1}{q_{2}}}\left(J, \mathbb{R}_{+}\right)$. For brevity, let $N:=\|\eta\|_{L^{\frac{1}{q_{2}}}(J)}$, $\gamma:=\frac{q-1}{1-q_{2}} \in(-1,0)$.

We introduce the following assumptions: 
(C1) $f: J^{4} \rightarrow \mathbb{R}$ is a Carathéodory function.

(C2) $f \in C\left(J^{4}, R\right)$ and there exist $L_{u}, L_{v}, L_{w}>0$ such that

$$
\left|f\left(t, u_{1}, v_{1}, w_{1}\right)-f\left(t, u_{2}, v_{2}, w_{2}\right)\right| \leq L_{u}\left|u_{1}-u_{2}\right|+L_{v}\left|v_{1}-v_{2}\right|+L_{w}\left|w_{1}-w_{2}\right|
$$

for all $t, u_{i}, v_{i}, w_{i} \in J, i=1,2$.

(C3) There are $m_{f}, M_{f}, 0<\theta \leq 1$ such that

$$
0 \leq m_{f}<M_{f} \leq \theta \Gamma(q+1)
$$

and

$$
m_{f} \leq f(t, u, v, w) \leq M_{f} \quad \text { for all } t \in J .
$$

(C4) There exists a constant $L>0$ such that $L \geq \frac{\max \left\{\left|m_{f}\right|,\left|M_{f}\right|\right\}}{\Gamma(q)}$.

(C5) For some $\tau>0$ and $0<q_{1}, \lambda<1$,

$$
L_{A}:=\frac{1}{\Gamma(q)}\left(\frac{1-q_{1}}{q-q_{1}}\right)^{1-q_{1}}\left(L_{u}+\frac{L_{v}+L_{w} L \lambda}{\lambda^{q_{1}}}+\frac{L_{w}}{\left(\lambda^{2} \theta\right)^{q_{1}}}\right)\left(\frac{q_{1}}{\tau}\right)^{q_{1}}<1 .
$$

Theorem 3.1 Assume that (C1)-(C5) are satisfied. Then problem (3) has a unique solution in $\left(C_{L, \theta}(J, J),\|\cdot\|_{B}\right)$.

Proof Consider the operator

$$
A:\left(C_{L, \theta}(J, J),\|\cdot\|_{B}\right) \rightarrow\left(C(J, \mathbb{R}),\|\cdot\|_{B}\right)
$$

defined by

$$
A(x)(t)=\frac{1}{\Gamma(q)} \int_{0}^{t}(t-s)^{q-1} f(s, x(s), x(\lambda s), x(\lambda x(\lambda s))) d s, \quad t \in J .
$$

It is clear that $\left(C_{L, \theta}(J, J),\|\cdot\|_{B}\right)$ is a nonempty bounded closed convex subset of the Banach space $\left(C(J, \mathbb{R}),\|\cdot\|_{B}\right)$.

We firstly prove that $C_{L, \theta}(J, J)$ is an invariant subset for $A$. In fact, we obtain $0 \leq A x(t) \leq 1$ and $A x(t) \leq \theta t$ for all $t \in J$ clearly due to (C2) and (C3).

Moreover, consider $0<s_{1}<s_{2} \leq 1$. Then

$$
\begin{aligned}
\left|A(x)\left(s_{2}\right)-A(x)\left(s_{1}\right)\right| & \\
\leq & \frac{1}{\Gamma(q)} \int_{0}^{s_{1}}\left|\left(s_{2}-s\right)^{q-1}-\left(s_{1}-s\right)^{q-1}\right||f(s, x(s), x(\lambda s), x(\lambda x(\lambda s)))| d s \\
& +\frac{1}{\Gamma(q)} \int_{s_{1}}^{s_{2}}\left(s_{2}-s\right)^{q-1}|f(s, x(s), x(\lambda s), x(\lambda x(\lambda s)))| d s \\
\leq & \frac{\max \left\{\left|m_{f}\right|,\left|M_{f}\right|\right\}}{\Gamma(q)}\left(\int_{0}^{s_{1}}\left[\left(s_{2}-s\right)^{q-1}-\left(s_{1}-s\right)^{q-1}\right] d s+\int_{s_{1}}^{s_{2}}\left(s_{2}-s\right)^{q-1} d s\right) \\
\leq & \frac{\max \left\{\left|m_{f}\right|,\left|M_{f}\right|\right\}}{\Gamma(q)}\left|s_{2}-s_{1}\right| .
\end{aligned}
$$


Moreover, for $0=s_{1}<s_{2} \leq 1$, we have

$$
\begin{aligned}
\left|A(x)\left(s_{2}\right)-A(x)\left(s_{1}\right)\right| & \leq\left|\frac{1}{\Gamma(q)} \int_{s_{1}}^{s_{2}}\left(s_{2}-s\right)^{q-1} f(s, x(s), x(\lambda s), x(\lambda x(\lambda s))) d s\right| \\
& \leq \frac{\max \left\{\left|m_{f}\right|,\left|M_{f}\right|\right\}}{\Gamma(q+1)}\left|s_{2}-s_{1}\right| .
\end{aligned}
$$

Thus, we have $C_{L, \theta}(J, J)$ is an invariant subset for the operator $A$.

From condition (C5) it follows that $A$ is a Picard mapping. Indeed, for all $t \in J$, we get

$$
\begin{aligned}
& \left|A\left(x_{1}\right)(t)-A\left(x_{2}\right)(t)\right| \\
& \leq \frac{1}{\Gamma(q)} \int_{0}^{t}(t-s)^{q-1}\left[L_{u}\left|x_{1}(s)-x_{2}(s)\right|+L_{v}\left|x_{1}(\lambda s)-x_{2}(\lambda s)\right|\right. \\
& \left.+L_{w}\left|x_{1}\left(\lambda x_{1}(\lambda s)\right)-x_{2}\left(\lambda x_{2}(\lambda s)\right)\right|\right] d s \\
& \leq \frac{1}{\Gamma(q)} \int_{0}^{t}(t-s)^{q-1}\left[L_{u}\left|x_{1}(s)-x_{2}(s)\right|+L_{v}\left|x_{1}(\lambda s)-x_{2}(\lambda s)\right|\right. \\
& +L_{w}\left|x_{1}\left(\lambda x_{1}(\lambda s)\right)-x_{1}\left(\lambda x_{2}(\lambda s)\right)\right| \\
& \left.+L_{w}\left|x_{1}\left(\lambda x_{2}(\lambda s)\right)-x_{2}\left(\lambda x_{2}(\lambda s)\right)\right|\right] d s \\
& \leq \frac{1}{\Gamma(q)} \int_{0}^{t}(t-s)^{q-1}\left[L_{u}\left|x_{1}(s)-x_{2}(s)\right|+L_{v}\left|x_{1}(\lambda s)-x_{2}(\lambda s)\right|\right. \\
& +L_{w} L\left|\lambda x_{1}(\lambda s)-\lambda x_{2}(\lambda s)\right| \\
& \left.+L_{w}\left|x_{1}\left(\lambda x_{2}(\lambda s)\right)-x_{2}\left(\lambda x_{2}(\lambda s)\right)\right|\right] d s \\
& \leq \frac{1}{\Gamma(q)} \int_{0}^{t}(t-s)^{q-1}\left[L_{u}\left|x_{1}(s)-x_{2}(s)\right| e^{-\tau s} e^{\tau s}\right. \\
& +L_{v}\left|x_{1}(\lambda s)-x_{2}(\lambda s)\right| e^{-\tau \lambda s} e^{\tau \lambda s}+L_{w} L \lambda\left|x_{1}(\lambda s)-x_{2}(\lambda s)\right| e^{-\tau \lambda s} e^{\tau \lambda s} \\
& \left.+L_{w}\left|x_{1}\left(\lambda x_{2}(\lambda s)\right)-x_{2}\left(\lambda x_{2}(\lambda s)\right)\right| e^{-\tau \lambda x_{2}(\lambda s)} e^{\tau \lambda x_{2}(\lambda s)}\right] d s \\
& \leq \frac{1}{\Gamma(q)}\left[L_{u} \int_{0}^{t}(t-s)^{q-1} e^{\tau s} d s+L_{v} \int_{0}^{t}(t-s)^{q-1} e^{\tau \lambda s} d s\right. \\
& \left.+L_{w} L \lambda \int_{0}^{t}(t-s)^{q-1} e^{\tau \lambda s} d s+L_{w} \int_{0}^{t}(t-s)^{q-1} e^{\tau \lambda x_{2}(\lambda s)} d s\right]\left\|x_{1}-x_{2}\right\|_{B} \\
& \leq \frac{1}{\Gamma(q)}\left[L_{u} \int_{0}^{t}(t-s)^{q-1} e^{\tau s} d s+L_{v} \int_{0}^{t}(t-s)^{q-1} e^{\tau \lambda s} d s\right. \\
& +L_{w} L \lambda \int_{0}^{t}(t-s)^{q-1} e^{\tau \lambda s} d s \\
& \left.+L_{w} \int_{0}^{t}(t-s)^{q-1} e^{\tau \lambda^{2} \theta s} d s\right]\left\|x_{1}-x_{2}\right\|_{B} \\
& \leq \frac{1}{\Gamma(q)}\left[L_{u}\left(\frac{1-q_{1}}{q-q_{1}}\right)^{1-q_{1}}\left(\frac{q_{1}}{\tau}\right)^{q_{1}} e^{t \tau}+L_{\nu}\left(\frac{1-q_{1}}{q-q_{1}}\right)^{1-q_{1}}\left(\frac{q_{1}}{\tau \lambda}\right)^{q_{1}} e^{t \tau \lambda}\right. \\
& +L_{w} L \lambda\left(\frac{1-q_{1}}{q-q_{1}}\right)^{1-q_{1}}\left(\frac{q_{1}}{\tau \lambda}\right)^{q_{1}} e^{t \tau \lambda} \\
& \left.+L_{w}\left(\frac{1-q_{1}}{q-q_{1}}\right)^{1-q_{1}}\left(\frac{q_{1}}{\tau \lambda^{2} \theta}\right)^{q_{1}} e^{t \tau \lambda^{2} \theta}\right]\left\|x_{1}-x_{2}\right\|_{B}
\end{aligned}
$$




$$
\begin{aligned}
\leq & \frac{1}{\Gamma(q)}\left(\frac{1-q_{1}}{q-q_{1}}\right)^{1-q_{1}}\left(\frac{q_{1}}{\tau}\right)^{q_{1}} e^{t \tau}\left[L_{u}+\frac{L_{v} e^{t \tau(\lambda-1)}}{\lambda q_{1}}\right. \\
& \left.+\frac{L_{w} L \lambda e^{t \tau(\lambda-1)}}{\lambda q_{1}}+\frac{L_{w} e^{t \tau\left(\lambda^{2} \theta-1\right)}}{\left(\lambda^{2} \theta\right)^{q_{1}}}\right]\left\|x_{1}-x_{2}\right\|_{B} \\
\leq & \frac{1}{\Gamma(q)}\left(\frac{1-q_{1}}{q-q_{1}}\right)^{1-q_{1}}\left(L_{u}+\frac{L_{v}+L_{w} L \lambda}{\lambda^{q_{1}}}+\frac{L_{w}}{\left(\lambda^{2} \theta\right)^{q_{1}}}\right)\left(\frac{q_{1}}{\tau}\right)^{q_{1}} e^{t \tau}\left\|x_{1}-x_{2}\right\|_{B},
\end{aligned}
$$

which yields that

$$
\begin{aligned}
& \left|A\left(x_{1}\right)(t)-A\left(x_{2}\right)(t)\right| e^{-\tau t} \\
& \quad \leq \frac{1}{\Gamma(q)}\left(\frac{1-q_{1}}{q-q_{1}}\right)^{1-q_{1}}\left(L_{u}+\frac{L_{v}+L_{w} L \lambda}{\lambda^{q_{1}}}+\frac{L_{w}}{\left(\lambda^{2} \theta\right)^{q_{1}}}\right)\left(\frac{q_{1}}{\tau}\right)^{q_{1}}\left\|x_{1}-x_{2}\right\|_{B} .
\end{aligned}
$$

Thus

$$
\begin{aligned}
& \left\|A\left(x_{1}\right)-A\left(x_{2}\right)\right\|_{B} \\
& \leq \frac{1}{\Gamma(q)}\left(\frac{1-q_{1}}{q-q_{1}}\right)^{1-q_{1}}\left(L_{u}+\frac{L_{v}+L_{w} L \lambda}{\lambda q_{1}}+\frac{L_{w}}{\left(\lambda^{2} \theta\right)^{q_{1}}}\right)\left(\frac{q_{1}}{\tau}\right)^{q_{1}}\left\|x_{1}-x_{2}\right\|_{B}
\end{aligned}
$$

where we use the inequality

$$
\begin{aligned}
\int_{0}^{t}(t-s)^{q-1} e^{\tau s} d s & \leq\left(\int_{0}^{t}(t-s)^{\frac{q-1}{1-q_{1}}} d s\right)^{1-q_{1}}\left(\int_{0}^{t} e^{\frac{\tau s}{q_{1}}} d s\right)^{q_{1}} \\
& \leq\left(\frac{1-q_{1}}{q-q_{1}} t^{\frac{q-q_{1}}{1-q_{1}}}\right)^{1-q_{1}}\left(\frac{q_{1}}{\tau}\left(e^{\frac{t \tau}{q_{1}}}-1\right)\right)^{q_{1}} \\
& \leq\left(\frac{1-q_{1}}{q-q_{1}}\right)^{1-q_{1}}\left(\frac{q_{1}}{\tau}\right)^{q_{1}}\left(e^{\frac{t \tau}{q_{1}}}\right)^{q_{1}} \\
& =\left(\frac{1-q_{1}}{q-q_{1}}\right)^{1-q_{1}}\left(\frac{q_{1}}{\tau}\right)^{q_{1}} e^{t \tau}
\end{aligned}
$$

So we get

$$
\left\|A\left(x_{1}\right)-A\left(x_{2}\right)\right\|_{B} \leq L_{A}\left\|x_{1}-x_{2}\right\|_{B}
$$

where

$$
L_{A}=\frac{1}{\Gamma(q)}\left(\frac{1-q_{1}}{q-q_{1}}\right)^{1-q_{1}}\left(L_{u}+\frac{L_{v}+L_{w} L \lambda}{\lambda^{q_{1}}}+\frac{L_{w}}{\left(\lambda^{2} \theta\right)^{q_{1}}}\right)\left(\frac{q_{1}}{\tau}\right)^{q_{1}} .
$$

Thus, $A$ is of Lipschitz type with a constant $L_{A} \in(0,1)$ due to (C5). By applying the contraction principle, we obtain that $A$ is a Picard operator. This completes the proof.

4 Second results in $\left(C_{L}(J, J),\|\cdot\|_{C}\right)$

We give the following necessary assumptions.

$\left(\mathrm{C1}^{\prime}\right)$ There are $m_{f}, M_{f}$ such that

$$
0 \leq m_{f}<M_{f} \leq \Gamma(q+1)
$$


and

$$
m_{f} \leq f(t, u, w) \leq M_{f} \quad \text { for all } t \in J
$$

$\left(\mathrm{C} 2^{\prime}\right)$ There is $0<\lambda<1$ such that $L_{A}^{\prime}:=\frac{L_{u}+L_{v}+L_{w}+L_{w} L \lambda}{\Gamma(q+1)}<1$.

Theorem 4.1 Assume that (C1), (C2), (C4), (C1') and (C2') are satisfied. Then problem (3) has a unique solution in $\left(C_{L}(J, J),\|\cdot\|_{C}\right)$.

Proof Consider the operator

$$
A:\left(C_{L}(J, J),\|\cdot\|_{C}\right) \rightarrow\left(C(J, \mathbb{R}),\|\cdot\|_{C}\right)
$$

defined by (4).

It is clear that $\left(C_{L}(J, J),\|\cdot\|_{C}\right)$ is a nonempty bounded closed convex subset of the Banach space $\left(C(J, \mathbb{R}),\|\cdot\|_{C}\right)$.

Step 1. According to (C1), (C2), (C4), $\left(\mathrm{C1}^{\prime}\right), C_{L}(J, J)$ is clearly an invariant subset for $A$.

Step 2. We prove that $A$ is of Lipschitz type with a constant

$$
L_{A}^{\prime}=\frac{L_{u}+L_{v}+L_{w}+L_{w} L \lambda}{\Gamma(q+1)} .
$$

Indeed, for all $t \in J$, taking into account (C1), we get

$$
\begin{aligned}
\left|A\left(x_{1}\right)(t)-A\left(x_{2}\right)(t)\right| & \\
\leq & \frac{1}{\Gamma(q)} \int_{0}^{t}(t-s)^{q-1}\left[L_{u}\left|x_{1}(s)-x_{2}(s)\right|+L_{v}\left|x_{1}(\lambda s)-x_{2}(\lambda s)\right|\right. \\
& \left.+L_{w}\left|x_{1}\left(\lambda x_{1}(\lambda s)\right)-x_{2}\left(\lambda x_{2}(\lambda s)\right)\right|\right] d s \\
\leq & \frac{1}{\Gamma(q)} \int_{0}^{t}(t-s)^{q-1}\left[L_{u}\left|x_{1}(s)-x_{2}(s)\right|+L_{v}\left|x_{1}(\lambda s)-x_{2}(\lambda s)\right|\right. \\
& \left.+L_{w}\left|x_{1}\left(\lambda x_{1}(\lambda s)\right)-x_{1}\left(\lambda x_{2}(\lambda s)\right)\right|+L_{w}\left|x_{1}\left(\lambda x_{2}(\lambda s)\right)-x_{2}\left(\lambda x_{2}(\lambda s)\right)\right|\right] d s \\
\leq & \frac{1}{\Gamma(q)} \int_{0}^{t}(t-s)^{q-1}\left[L_{u}\left\|x_{1}-x_{2}\right\|_{C}+L_{v}\left\|x_{1}-x_{2}\right\|_{C}\right. \\
& \left.+L_{w} L\left|\lambda x_{1}(\lambda s)-\lambda x_{2}(\lambda s)\right|+L_{w}\left\|x_{1}-x_{2}\right\|_{C}\right] d s \\
\leq & \frac{1}{\Gamma(q)} \int_{0}^{t}(t-s)^{q-1}\left[L_{u}\left\|x_{1}-x_{2}\right\|_{C}+L_{v}\left\|x_{1}-x_{2}\right\|_{C}\right. \\
& \left.+L_{w} L \lambda\left\|x_{1}-x_{2}\right\|_{C}+L_{w}\left\|x_{1}-x_{2}\right\|_{C}\right] d s \\
\leq & \frac{L_{u}+L_{v}+L_{w}+L_{w} L \lambda}{\Gamma(q+1)}\left\|x_{1}-x_{2}\right\|_{C} \\
= & L_{A}^{\prime}\left\|x_{1}-x_{2}\right\|_{C} .
\end{aligned}
$$

So we get

$$
\left\|A\left(x_{1}\right)-A\left(x_{2}\right)\right\|_{C} \leq L_{A}^{\prime}\left\|x_{1}-x_{2}\right\|_{C} .
$$


Thus, $A$ is of Lipschitz type with a constant $L_{A}^{\prime} \in(0,1)$ due to $\left(\mathrm{C} 2^{\prime}\right)$. By applying the contraction principle, we obtain that $A$ is a Picard operator. This completes the proof.

\section{Third results in $\left(C_{L}(J, J),\|\cdot\|_{\beta}\right)$}

We impose the following condition:

$\left(\mathrm{C} 1^{\prime \prime}\right)$ There is $0<\lambda<1$ such that $L_{A}^{\prime \prime}:=\frac{L_{u}^{\beta}+L_{v}^{\beta}+\left(L_{w} L \lambda\right)^{\beta}+L_{w}^{\beta}}{\Gamma^{\beta}(q+1)}<1$.

Theorem 5.1 Assume that (C1), (C2), (C4), (C1'), (C1") are satisfied. Then problem (3) has a unique solution in $\left(C_{L}(J, J),\|\cdot\|_{\beta}\right)$.

Proof Consider $A:\left(C_{L}(J, J),\|\cdot\|_{\beta}\right) \rightarrow\left(C(J, R),\|\cdot\|_{\beta}\right)$ given by (4).

It is clear that $\left(C_{L}(J, J),\|\cdot\|_{\beta}\right)$ is a nonempty bounded closed convex subset of the Banach space $\left(C(J, R),\|\cdot\|_{\beta}\right)$.

Similar to the proof of Step 1 in Theorem 3.1, one can easily verify that $C_{L}(J, J)$ is an invariant subset for $A$ due to (C1), (C2), (C4), (C1').

Next, we have to prove that $A$ is a Lipschitz-type operator.

For all $x, z \in C_{L}(J, J)$, by using our conditions, we have

$$
\begin{aligned}
& |A(x)(t)-A(z)(t)|^{\beta} \\
& \leq\left(\frac{1}{\Gamma(q)}\right)^{\beta} \mid \int_{0}^{t}(t-s)^{q-1}\left[L_{u}\left|x_{1}(s)-x_{2}(s)\right|+L_{v}\left|x_{1}(\lambda s)-x_{2}(\lambda s)\right|\right. \\
& \left.+L_{w}\left|x_{1}\left(\lambda x_{1}(\lambda s)\right)-x_{2}\left(\lambda x_{2}(\lambda s)\right)\right|\right]\left.d s\right|^{\beta} \\
& \leq\left(\frac{1}{\Gamma(q)}\right)^{\beta} \mid \int_{0}^{t}(t-s)^{q-1}\left[L_{u}\left|x_{1}(s)-x_{2}(s)\right|+L_{v}\left|x_{1}(\lambda s)-x_{2}(\lambda s)\right|\right. \\
& \left.+L_{w}\left|x_{1}\left(\lambda x_{1}(\lambda s)\right)-x_{1}\left(\lambda x_{2}(\lambda s)\right)\right|+L_{w}\left|x_{1}\left(\lambda x_{2}(\lambda s)\right)-x_{2}\left(\lambda x_{2}(\lambda s)\right)\right|\right]\left.d s\right|^{\beta} \\
& \leq\left(\frac{1}{\Gamma(q)}\right)^{\beta} \mid \int_{0}^{t}(t-s)^{q-1}\left[L_{u}\left|x_{1}(s)-x_{2}(s)\right|+L_{v}\left|x_{1}(\lambda s)-x_{2}(\lambda s)\right|\right. \\
& \left.+L_{w} L \lambda\left|x_{1}(\lambda s)-x_{2}(\lambda s)\right|+L_{w}\left|x_{1}\left(\lambda x_{2}(\lambda s)\right)-x_{2}\left(\lambda x_{2}(\lambda s)\right)\right|\right]\left.d s\right|^{\beta} \\
& \leq\left(\frac{1}{\Gamma(q)}\right)^{\beta} \mid \int_{0}^{t}(t-s)^{q-1}\left[L_{u}\left\|x_{1}-x_{2}\right\|_{\beta}^{\frac{1}{\beta}}+L_{v}\left\|x_{1}-x_{2}\right\|_{\beta}^{\frac{1}{\beta}}\right. \\
& \left.+L_{w} L \lambda\left\|x_{1}-x_{2}\right\|_{\beta}^{\frac{1}{\beta}}+L_{w}\left\|x_{1}-x_{2}\right\|_{\beta}^{\frac{1}{\beta}}\right]\left.d s\right|^{\beta} \\
& \leq \frac{1}{\Gamma^{\beta}(q+1)}\left[L_{u}^{\beta}\left\|x_{1}-x_{2}\right\|_{\beta}+L_{v}^{\beta}\left\|x_{1}-x_{2}\right\|_{\beta}\right. \\
& \left.+\left(L_{w} L \lambda\right)^{\beta}\left\|x_{1}-x_{2}\right\|_{\beta}+L_{w}^{\beta}\left\|x_{1}-x_{2}\right\|_{\beta}\right] \\
& \leq \frac{L_{u}^{\beta}+L_{v}^{\beta}+\left(L_{w} L \lambda\right)^{\beta}+L_{w}^{\beta}}{\Gamma^{\beta}(q+1)}\left\|x_{1}-x_{2}\right\|_{\beta}
\end{aligned}
$$

where we use the inequality $(a+b)^{n} \leq a^{n}+b^{n}$ for any $n<1$ and nonnegative $a, b$. 
So we get

$$
\left\|A\left(x_{1}\right)-A\left(x_{2}\right)\right\|_{\beta} \leq L_{A}^{\prime \prime}\left\|x_{1}-x_{2}\right\|_{\beta}
$$

Thus, $A$ is of Lipschitz type with a constant $L_{A}^{\prime \prime} \in(0,1)$ due to $\left(\mathrm{C}^{\prime \prime}\right)$. By applying the contraction principle, we obtain that $A$ is a Picard operator. This completes the proof.

\section{Example}

Let us consider the following problem:

$$
\left\{\begin{array}{l}
{ }^{c} D_{0, t}^{1.5} x(t)=0.5 x(0.5 x(0.5 t)), \quad t \in[0,1] \\
x(0)=0, \quad x^{\prime}(0)=0
\end{array}\right.
$$

We have the following two propositions.

Proposition 6.1 Problem (5) has a unique solution in $\left(C_{0.56419}([0,1],[0,1]),\|\cdot\|_{C}\right)$.

Proof By Theorem 4.1, we choose $\lambda=\frac{1}{2}, q=\frac{3}{2}, L=0.56419, m_{f}=0$ and $M_{f}=\frac{1}{2}$. Clearly, one can verify that conditions $(\mathrm{C} 1),(\mathrm{C} 2),(\mathrm{C} 4),\left(\mathrm{C1}^{\prime}\right)$ and $\left(\mathrm{C}^{\prime}\right)$ from Theorem 4.1 hold.

Proposition 6.2 Problem (5) has a unique solution in $\left(C_{0.56419}([0,1],[0,1]),\|\cdot\|_{\frac{1}{2}}\right)$.

Proof By Theorem 5.1, we choose $\beta=\frac{1}{2}, \lambda=\frac{1}{2}, q=\frac{3}{2}, L=0.56419, m_{f}=0$ and $M_{f}=\frac{1}{2}$. Clearly, one can verify that conditions (C1), (C2), (C4), $\left(\mathrm{C1}^{\prime}\right)$ and $\left(\mathrm{C1}^{\prime \prime}\right)$ from Theorem 5.1 hold.

\footnotetext{
Competing interests

The authors declare that they have no competing interests
}

\section{Authors' contributions}

This work was carried out in collaboration between all authors. JRW raised these interesting problems in this research. JRW and JHD proved the theorems, interpreted the results and wrote the article. All authors defined the research theme, read and approved the manuscript.

\footnotetext{
Acknowledgements

The authors thank the referees for their careful reading of the manuscript and insightful comments, which helped to improve the quality of the paper. We would also like to acknowledge the valuable comments and suggestions from the editors, which vastly contributed to improving the presentation of the paper. This work is supported by the National Natural Science Foundation of China (11201091), Key Projects of Science and Technology Research in the Chinese Ministry of Education (211169), Key Support Subject (Applied Mathematics) and Key project on the reforms of teaching contents and course system of Guizhou Normal College.

Received: 3 July 2013 Accepted: 15 October 2013 Published: 19 Nov 2013

References

1. Kelley, CT: Iterative Methods for Linear and Nonlinear Equations. Society for Industrial and Applied Mathematics, Philadelphia (1995)

2. Wang, K: In the equation $x^{\prime}(t)=f(x(x(t)))$. Funkc. Ekvacioj 33, 405-425 (1990)

3. Medved, M: On minimal periods of functional-differential equations and difference inclusions. Ann. Pol. Math. LIV(3), 263-270 (1991)

4. Fečkan, M: On a certain type of functional differential equations. Math. Slovaca 43, 39-43 (1993)

5. Si, J, Wang, Xi: Smooth solutions of a nonhomogeneous iterative functional differential equations with variable coefficients. J. Math. Anal. Appl. 226, 377-392 (1998)

6. Staněk, S: Global properties of solutions of iterative-differential equations. Funct. Differ. Equ. 5, 463-481 (1998)
} 
7. Liu, J: Analytic solutions of a first order iterative functional differential equation. Results Math. 55, 129-137 (2009)

8. Egri, E, Rus, IA: First order iterative functional-differential equation with parameter. Stud. Univ. Babeş-Bolyai, Math. LII, 67-80 (2007)

9. Mureşan, V: Volterra integral equations with iterations of linear modification of the argument. Novi Sad J. Math. 33, 1-10 (2003)

10. Baleanu, D, Machado, JAT, Luo, ACJ (eds.): Fractional Dynamics and Control. Springer, New York (2012)

11. Diethelm, K: The Analysis of Fractional Differential Equations. Lecture Notes in Mathematics. Springer, Berlin (2010)

12. Kilbas, AA, Srivastava, HM, Trujillo, JJ: Theory and Applications of Fractional Differential Equations. Elsevier, Amsterdam (2006)

13. Lakshmikantham, V, Leela, S, Devi, JV: Theory of Fractional Dynamic Systems. Cambridge Scientific Publishers, Cambridge (2009)

14. Miller, KS, Ross, B: An Introduction to the Fractional Calculus and Differential Equations. Wiley, New York (1993)

15. Michalski, MW: Derivatives of noninteger order and their applications. Diss. Math. CCCXXVIII, 1-47 (1993)

16. Podlubny, I: Fractional Differential Equations. Academic Press, San Diego (1999)

17. Tarasov, VE: Fractional Dynamics: Application of Fractional Calculus to Dynamics of Particles, Fields and Media. Springer, Berlin (2011)

18. Wang, J, Fečkan, M, Zhou, Y: Fractional order iterative functional differential equations with parameter. Appl. Math Model. 37, 6055-6067 (2013)

19. Li, X, Chen, F, Li, X: Generalized anti-periodic boundary value problems of impulsive fractional differential equations. Commun. Nonlinear Sci. Numer. Simul. 18, 28-41 (2013)

20. Rus, IA: Functional-differential equations of mixed type, via weakly Picard operators. Semin. Fixed Point Theory Cluj-Napoca 3, 335-345 (2002)

21. Rus, IA: Picard operators and applications. Sci. Math. Jpn. 58, 191-219 (2003)

22. Rus, IA: Picard mappings: results and problems. Preprint. Cluj-Napoca: Babeş-Bolyai Univ., Seminar on Fixed Point Theory, Preprint 6, 55-64 (1987)

23. Rus, IA: Weakly Picard mappings. Comment. Math. Univ. Carol. 34, 769-773 (1993)

24. Jung, SM, Kim, TS, Lee, KS: A fixed point approach to the stability of quadratic functional equation. Bull. Korean Math. Soc. 43, 531-541 (2006)

10.1186/1687-1847-2013-329

Cite this article as: Wang and Deng: Fractional order differential equations with iterations of linear modification of the argument. Advances in Difference Equations 2013, 2013:329

\section{Submit your manuscript to a SpringerOpen ${ }^{\circ}$ journal and benefit from:}

- Convenient online submission

Rigorous peer review

- Immediate publication on acceptance

Open access: articles freely available online

- High visibility within the field

- Retaining the copyright to your article 